\title{
The development and origins of vertebrate meninges
}

\section{Munirah Batarfi ${ }^{1,2}$, Petr Valasek ${ }^{1,3}$, Eliska Krejci ${ }^{3}$, Ruijin Huang ${ }^{4,5}$, and Ketan Patel ${ }^{1,6}$}

1 School of Biological Sciences, University of Reading,

Hopkins Building, Reading, RG6 6UB, UK; ketan.patel@reading.ac.uk

${ }^{2}$ Anatomy department, Faculty of medicine, King Fahad Medical City, Riyadh, Saudi Arabia

3 Institute of Anatomy, First Faculty of Medicine, Charles University,

U Nemocnice 3, Praha 2, Prague, 128 00, Czech Republic

4 Department of Neural Anatomy, Institute of Anatomy, University of Bonn, Germany

5 Department of Molecular Embryology, Institute of Anatomy and Cell Biology,

University of Freiburg, Germany

${ }^{6}$ Freiburg Institute for Advanced Studies (FRIAS), University of Freiburg, Freiburg, Germany

Address correspondence and requests for materials to Ketan Patel

\begin{abstract}
Meninges comprise three distinct layers, the dura mater, arachnoid, and pia mater that surround the brain, spinal cord and some parts of the nerves. Traditionally the meninges were believed to serve only as protection for tissues that they encase. However recent work shows they have other important functions related to development and regulation of the nervous system. Given the importance of the meninges, it is surprising that we know very little about their development. The embryological origin of the meninges has been debated for over a hundred years. Some studies imply that the meninges develop from the neural crest, while others suggest that they come from the somites. Here, we investigated the temporal development of meninges in birds and mice and found they form at comparable stages. We investigated the origin of avian spinal meninges using chick/quail cell tracing protocols and found they do not develop from the somites as previously thought. We propose that meningeal epithelial blood vessels may have been mistaken as meninges and led to an erroneous conclusion by previous investigators. We present data that show that avian spinal meninges originated from the neural crest supported by data demonstrating that they express the neural crest marker HNK1. Finally using the Wnt1-Cre mouse we show that trunk meninges of mammals also originate from neural crest.
\end{abstract}

Citation: Batarfi, M., Valasek, P., Krejci, E., Huang, R., and Patel, K. 2017. The development and origins of vertebrate meninges. Bio. Comm. 62(2): 73-81. doi: $10.21638 / 11701 /$ spbu03.2017.203

Author's information: Dr. Munirah Batarfi, postdoctoral fellow, orcid.org/0000-00018750-1446; Dr. Petr Valasek, Doc. MUDr., MRCGP (Member of Royal College of General Practitioners), orcid.org/0000-00019224-4560; Dr. Eliska Krejci, orcid.org/00000002-3394-9971; Professor Ruijing Huang, orcid.org/0000-0003-0467-2907; Professor Ketan Patel, orcid.org/0000-0002-7131-749X

Manuscript Editor: Dunja Knapp, DFGCenter for Regenerative Therapies Dresden, Cluster of Excellence / TU Dresden,

Germany

Received: May 08, 2017;

Revised: May 23, 2017;

Accepted: June 01, 2017;

Copyright: (c) 2017 Batarfi et al. This is an open-access article distributed under the terms of the License Agreement with Saint Petersburg State University, which permits to the authors an unrestricted distribution and self-archiving free of charge.

Funding: King Fahad Medical City awarded a PhD scholarship to Munirah Batarfi.

Competing interests: The authors have declared that no competing interests exist.
Keywords: embryo, chick, mouse, meninges, development

\section{Introduction}

Meninges are the protective layers enveloping the brain, spinal cord and parts of the nerves. They offer protection and are also essential for central nervous system development. Meninges in mammals consist of three tissue layers: dura mater, arachnoid layer and pia mater. The dura mater (ectomeninx) is the thickest and the densest of the three connective tissue layers. The cranial dura is continuous with the spinal dura. It consists of two layers; the outer thicker layer called the endosteal that contains larger number of cells and blood vessels and the inner thinner layer called the meningeal layer. The dura is continuous with the periosteal layer of the orbit and with the pericranium through the suture of the skull (Clarke, 1944). The dura mater has been strongly implicated in the development of the skull (Richtsmeier and Flaherty, 2013). The arachnoid is an avascular connective tissue layer containing collagen and elastic fibres. It also contains fibroblasts and is covered by epithelial cells that are connected to each other by tight junctions (Haines, Harkey, and al-Mefty, 1993). The external surface of the arachnoid layer contains the arachnoid granulation and villi, which aid cerebral spi- 
nal fluid (CSF) circulation (Kapoor, Katz, Grzybowski, and Lubow, 2008). The pia mater is the deepest layer and the most difficult to identify, because of its delicate nature and adherence to the surface of the cerebral cortex (glia limitans) and spinal cord (Standring, Ellis, and Wigley, 2005). Its external surface is covered by epithelial cells that lack tight junctions. Both the arachnoid and pia mater contain elastic fibres network, integrated collagen bundles and large number of fibroblasts and macrophages. The pia mater also contains mast cells and lymphocytes in the perivascular areas (Bloom and Fawcett, 1968). The subarachnoid space lies between the arachnoid and pia mater and contains the CSF that starts to circulate at the fifth week of human embryonic life.

Meninges not only offer protection to the tissues they encapsulate but new work shows that they are essential for neural tissue development. For example, meninges secrete the chemokine Cxcl12 which enhances the movement of Cajal-Retzius (CR) cells along the cortical surface (Borrell and Marin, 2006). Additionally the pial basement membrane functions as an anchor for the radial glial cells (RGC) which are progenitors for neurons and glia, and their processes serve as guideposts for migrating neurons (Radakovits, Barros, Belvindrah, Patton, and Muller, 2009). Survival of RGCs and ultimately cortical size is dependent on the expression of laminins in the pia basement membrane (Radakovits et al., 2009). Remarkably recent work has even demonstrated that meninges are a niche for neural precursor cells (Bifari et al., 2015; Petricevic et al., 2011).

Given that meninges play a number of crucial roles associated with the central nervous system, it is a surprise that we have only a sketchy idea regarding their development. The embryonic origin of the meninges has been debated for over a hundred years with two candidate tissues being proposed to have this role: neural crest and the somites. O'Rahilly and Muller suggest that meninges of the head and body are of mixed origin (mesoderm and neural crest) (O’Rahilly and Muller, 1986). In contrast other studies have suggested that head meninges develop from neural crest (Jiang, Iseki, Maxson, Sucov, and Morriss-Kay, 2002; Korn, Christ, and Kurz, 2002) whereas body meninges are somitic in origin (Bagnall, Higgins, and Sanders, 1989; Christ, Huang, and Scaal, 2007; Halata, Grim, and Christ, 1990; Nimmagadda, Loganathan, Wilting, Christ, and Huang, 2004). Furthermore, it is surprising that there is no detailed study documenting the temporal development of the meninges in two extensively studied experimental animal models: the chick and mouse.

In this report we performed a detailed histological survey of meningeal development during chick and mouse embryogenesis. Secondly, given the canonical view that head and body meninges develop from differing origins we investigated their contribution at their transitional point; the neck. We have previously shown that this evolutionary vertebrate novelty is a region with unique developmental properties, for example the musculature originates from lateral plate mesoderm (Theis et al., 2010).

We report that the development of meninges in mouse and chick take place at comparable developmental stages. Our chick/quail chimera studies show that the somites make a huge contribution to the tissues that surround the developing neural tube. However the somitic cells formed vasculature tissue. Lineage tracing studies show that cells of neural tube form the meninges in both the neck and trunk region. In contrast to the somites, neural tube cells do not form blood vessels at the stages examined in our study. We therefore demonstrate that meninges of the neck and trunk are neural crest in origin, a notion supported by their expression of HNK1. Finally we used a genetic approach to show that neural crest forms mouse trunk meninges.

\section{Methods}

\section{CHICK AND MOUSE EMBRYOS STAGE SERIES COLLECTION}

Fertilised white leghorn chicken (Gallus gallus) eggs were purchased from (Henry Stewart poultry LTD UK) and incubated at $39^{\circ}$ in $80 \%$ humidity. All embryos were staged according to Hamburger and Hamilton (Hamburger and Hamilton, 1951) and harvested between HH16 to HH36 (D10). Transgenic and wild-type C57Bl6 mice were bred in house. Dissected embryos were fixed for one hour in 4\% PFA and stored in $1 \%$ PFA/PBS until processing for paraffin sectioning.

\section{PROCESSING OF TISSUE FOR MICROTOME SECTIONING}

Embryos were dehydrated in an ethanol series. Embryos were then incubated twice in xylene for $45 \mathrm{~min}$. Thereafter the embryos were submerged in molten wax for 1 hour at $65^{\circ} \mathrm{C}$. Finally, the embryos were embedded in a fresh wax. The embryos were sectioned at $10 \mu \mathrm{m}$ using microtome (MICROM HM360) and stained with Hematoxylin and Eosin or used for immunohistochemistry. QCPN (1:1 DSHB, Iowa, USA) and QH1 (1:10 DSHB, Iowa, USA) were detected using the $\mathrm{ABC}$ peroxidase staining protocol (Thermo Fisher UK).

\section{INDIAN INK INJECTION}

For Indian ink injection glass capillaries $(50 \mu \mathrm{m})$ were pulled using a standard needle puller. The Indian ink was diluted in PBS 1:5 and loaded in the glass capillaries. The chick embryos were exposed by removing the ectoderm with an electrically sharpened tungsten needle. The same tungsten needle was used to make a hole at the lower part of the dorsal aorta and used to inject the ink 
Cervical
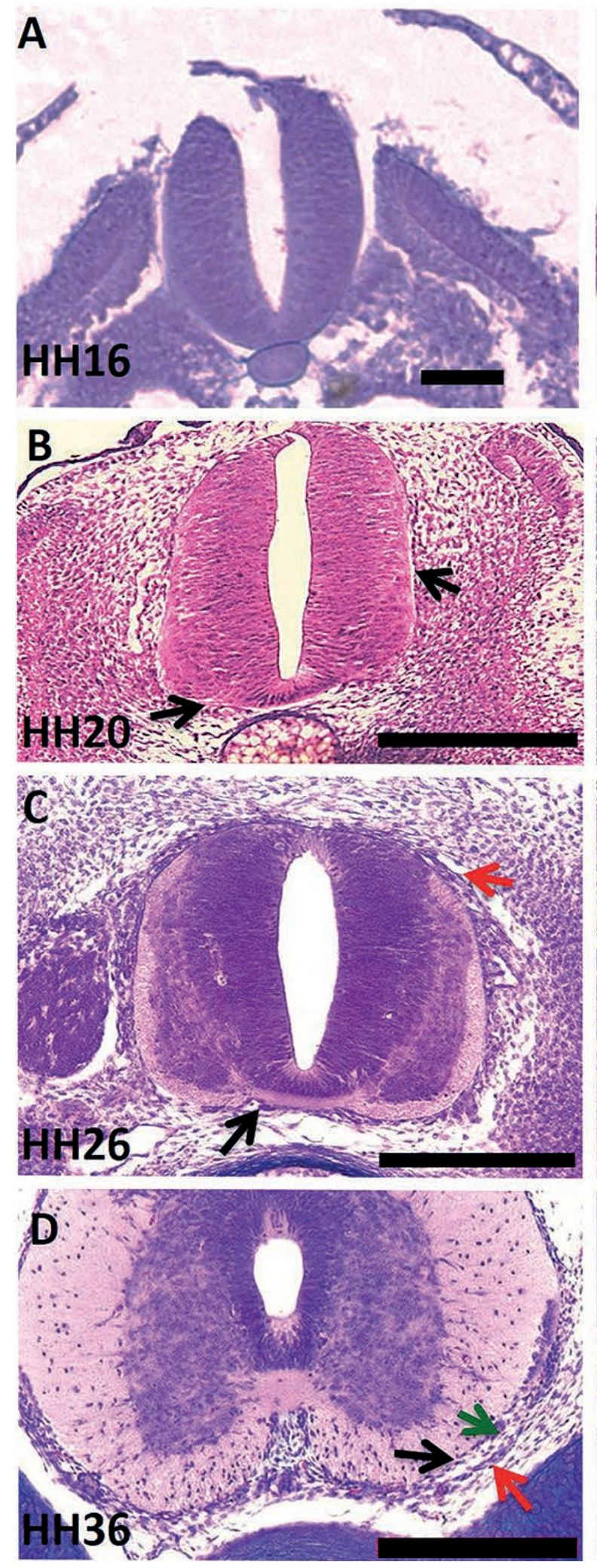

Thoracic
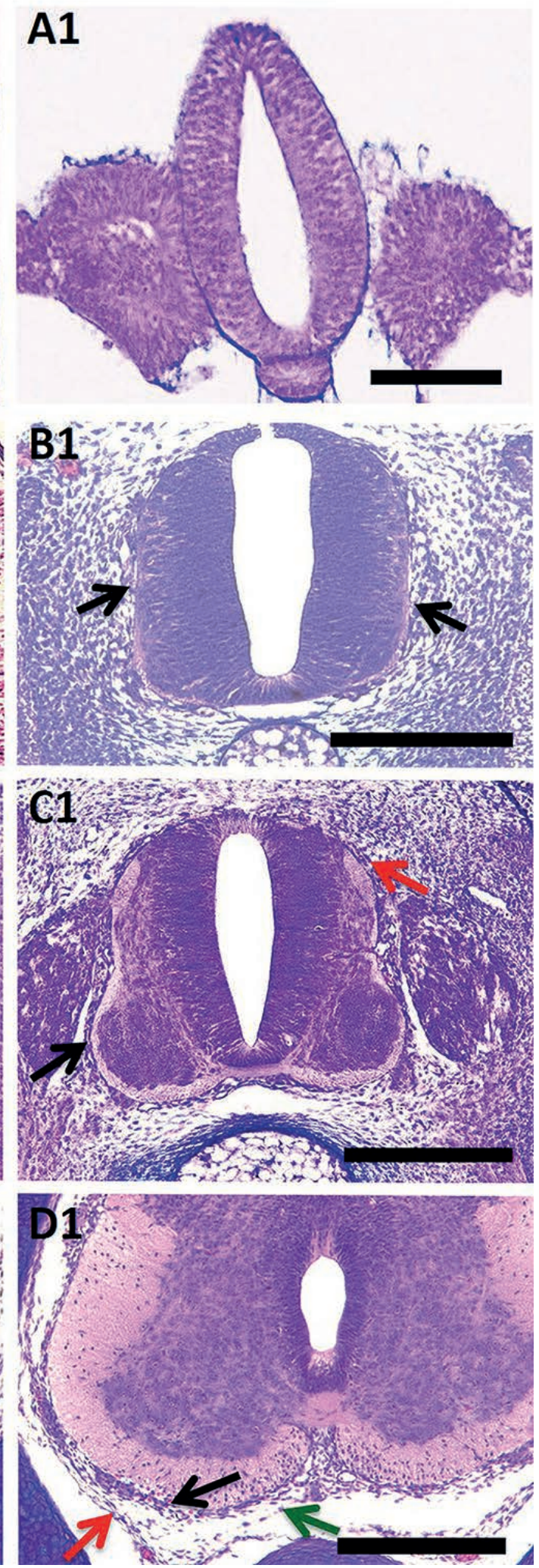

Fig. 1. Development of chick meninges.

(A-A1) $\mathrm{HH} 16$ section showing the neural tube and the somites. Note absence of meningeal tissue around the neural tube at cervical or thoracic levels. (B-B1) HH20 section showing a single meningeal layer in the perineural space (black arrow). Note the ventral location of the tissue. (C-C1) HH26 meningeal tissue located at the ventral (black arrows) and dorsal aspect (red arrow) of the neural tube. (D-D1) HH36 section showing three layers of meninges at cervical and thoracic level. Dense innermost latey adjacent to neural tube (black arrows), a looser cell layer in middle (green arrow) and more ordered outer layer (red arrow). Scale bars $-50 \mu \mathrm{M}$. 
into the circulation of the embryo. After robust perfusion, embryos were fixed in Serra fixative.

\section{QUAIL-CHICK TRANSPLANTATION}

The fertile chick (Gallus gallus domesticus) and quail (Coturnix coturnix japonica) (Brian Potter UK) eggs were incubated at $39^{\circ} \mathrm{C}$ in $80 \%$ humidity until stage HH10-12 (Hamburger and Hamilton, 1951). Donor tissue was prepared using the Chapman easy culture method (Chapman, Collignon, Schoenwolf, and Lumsden, 2001). Grafting of tissues was performed as previously described (Theis et al., 2010). Operated embryos were harvested at day 8 of embryonic life and fixed in Serra fixative.

\section{Wht-1Cre/R26R TISSUE}

Wnt-1Cre/R26R embryos were harvested E13.5, fixed for three hours in $4 \% \mathrm{PFA}$ in PBS then washed in PBS passed through a sucrose gradient and embedded in OTC. The embryos were sectioned at $7 \mu \mathrm{m}$ and were washed in PBS. Endogenous peroxidase was inactivated with $0.6 \% \mathrm{H}_{2} \mathrm{O}_{2}$ in PBS for 30 minutes. Non-specific binding was blocked with $10 \%$ goat serum for one hour. Rabbit polyclonal anti-beta-galactosidase antibody (1:800 Abcam ab616) was used overnight at $4{ }^{\circ} \mathrm{C}$. Laminin was detected with a mouse anti-laminin monoclonal antibody (1:200 Sigma L8271).

\section{Results}

\section{DEVELOPMENT OF CHICK MENINGES}

We started investigating the temporal development of chick meninges by examining HH16 embryos. At this stage there was no evidence of meningeal tissues at either the cervical or thoracic regions (Fig. 1A-A1). However at $\mathrm{HH} 20$ the meninges were evident predominantly at the ventral aspect of the neural tube at both cervical and thoracic levels (Fig. 1B-B1). The development of the meninges expanded dorsally so that by $\mathrm{HH} 26$, they were evident at the dorsal aspect of the neural tube (Fig. 1CC1). By HH36 three meningeal layers were evident at both levels (Fig. 1D-D1). The inner and outmost layers were denser compared with the middle layer.

\section{DEVELOPMENT OF MOUSE MENINGES}

The meninges were not evident at E9.5 at either the cervical or the thoracic level (Fig. 2A-A1). However at E10.5 a fine layer between the somite and the neural tube was evident at both levels (Fig. 2B-B1). Of note was the observation that the meninges were most prominent at lateral aspect of the neural tube. By E11.5 the meninges extended to surround the entire neural tube at both levels (Fig. 2C-C1). Finally, three layers were evident at
E15.5 (Fig. 2D-D1). As in the chick, the mouse meninges at this time had differing cellular organisation with the inner and outer being more compact and the middle layer made of loose connective tissue (Fig. 2D-D1). The zone between the inner and outer meningeal layers was invested with blood vessels (Fig. 2D).

\section{NEURAL CREST ORIGIN OF CHICK MENINGES}

Previous research suggested that there are two sources of meninges in vertebrates; the neural crest in the head and somites in the trunk (Halata et al., 1990; Jiang et al., 2002). Our original intention was to determine the origin of the meninges of the neck since it lies between these two regions. We therefore lineage traced somitic tissues using unilateral homotopic quail/chick chimeras. To that end we grafted somite 10 for the neck region and somite 22 for the thoracic. The results presented here are from the analysis of 11 surviving chimeras/embryos at the cervical regions and 26 for the thoracic. All 11 chimeras from the neck region operations showed incorporation of quail tissue. For the thoracic region the number was 18 . We found that cells of somitic origin were present in regions surrounding the neural tube in 8/11 neck and 18/18 thoracic embryos. Three neck and 5 thoracic chimeras were thereafter examined in detail by sectioning the entire embryo (Fig. $3 \mathrm{~A}$ and $\mathrm{B}$ ). In addition, we were surprised to find somitic cells in the neural tube and therefore investigated whether they were blood vessels. Immunohistochemistry using the quail specific endothelial marker, QH1 indeed confirmed the presence of blood vessels of somitic origin in the neural tube. However these sections showed extensive blood vessel development that was somitic in origin in the meningeal region (Fig. 3A1 and B1). We were only able to find somitic cells in the meninges that co-localised to $\mathrm{QH} 1$ expression. These results suggest that somitic cells form the vasculature of the neural tube. To confirm that the vasculature was indeed present at the stages under investigation, we injected Indian ink into the developing chick embryo to identify blood vessels. Our studies showed that the pattern of blood vessel distribution in the meninges and the neural tube was similar to the blood vessels originating from the grafted quail somites (Fig. 3C, C1).

These results show that somitic cells contribute to the formation of the vasculature associated with the meninges.

Having dismissed the somites as the origin of the meninges in either the neck or trunk region, we then examined the neural tube as a potential source. To that end we grafted neural tube at the level of somites 9-10 for the neck region and at the level of somite 20-22 for the thoracic as a control. The results presented are from the analysis of 4 fully incorporated graft operations at 


\section{Cervical}
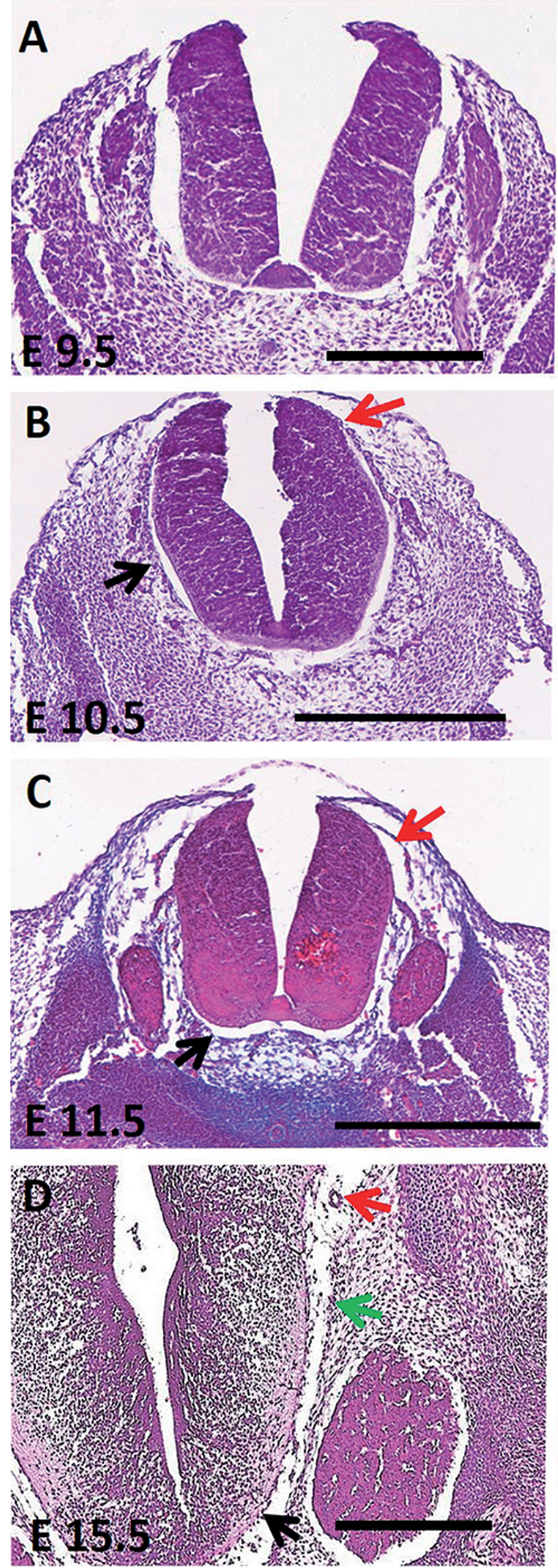

\section{Thoracic}
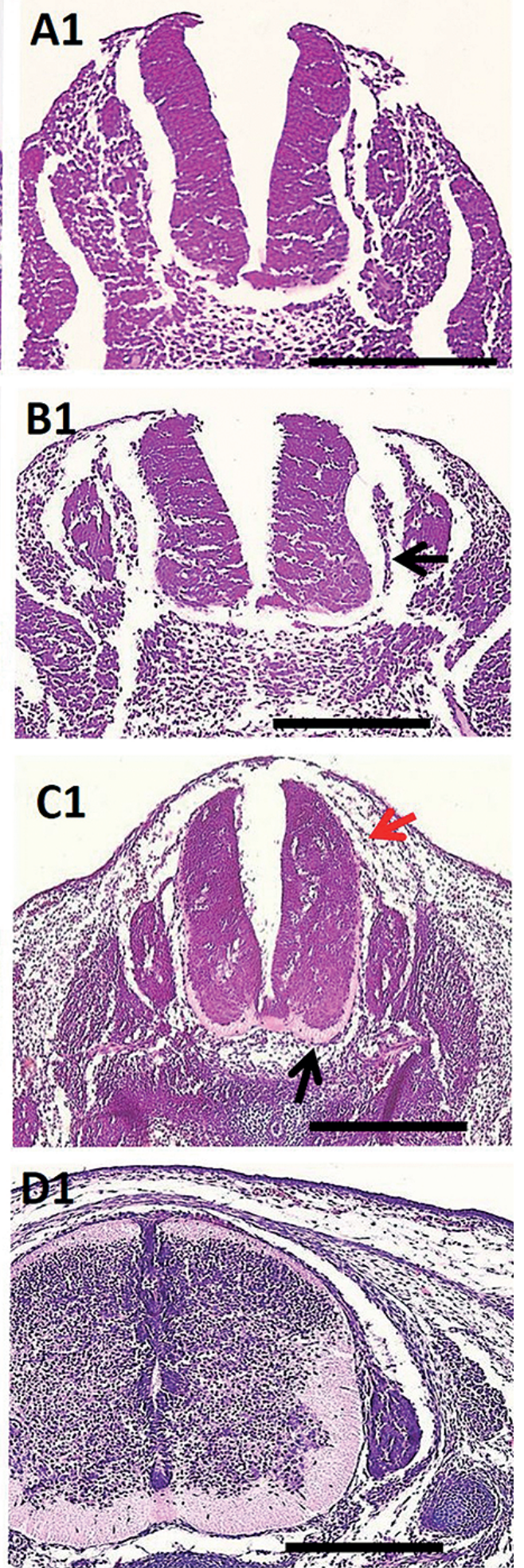

Fig. 2. Development of mouse meninges.

(A-A1) E9.5 section showing the neural tube and the somites. No meningeal layer identifiable at either cervical or thoracic level. (B-B1) E10.5 section showing a cellular layer at the lateral aspect of the neural tube at both cervical and thoracic levels. At the cervical level the meninges were evident at the dorsal aspect of the neural tube (red arrow) whereas they were less prominent at the ventral. In the thoracic region meninges were not present at the dorsal aspect of the neural tube. (C-C1) E11.5 section showing meninges at both dorsal (red arrow) and ventral aspect of the neural tube in cervical and thoracic level. (D-D1) E15.5 section showing a dense meningeal layer adjacent to the neural tube (black arrow) with a looser middle layer containing blood vessels (red arrow) and an outer more dense layer (green arrow). Scale bars - 50 $\mu \mathrm{M}$. 


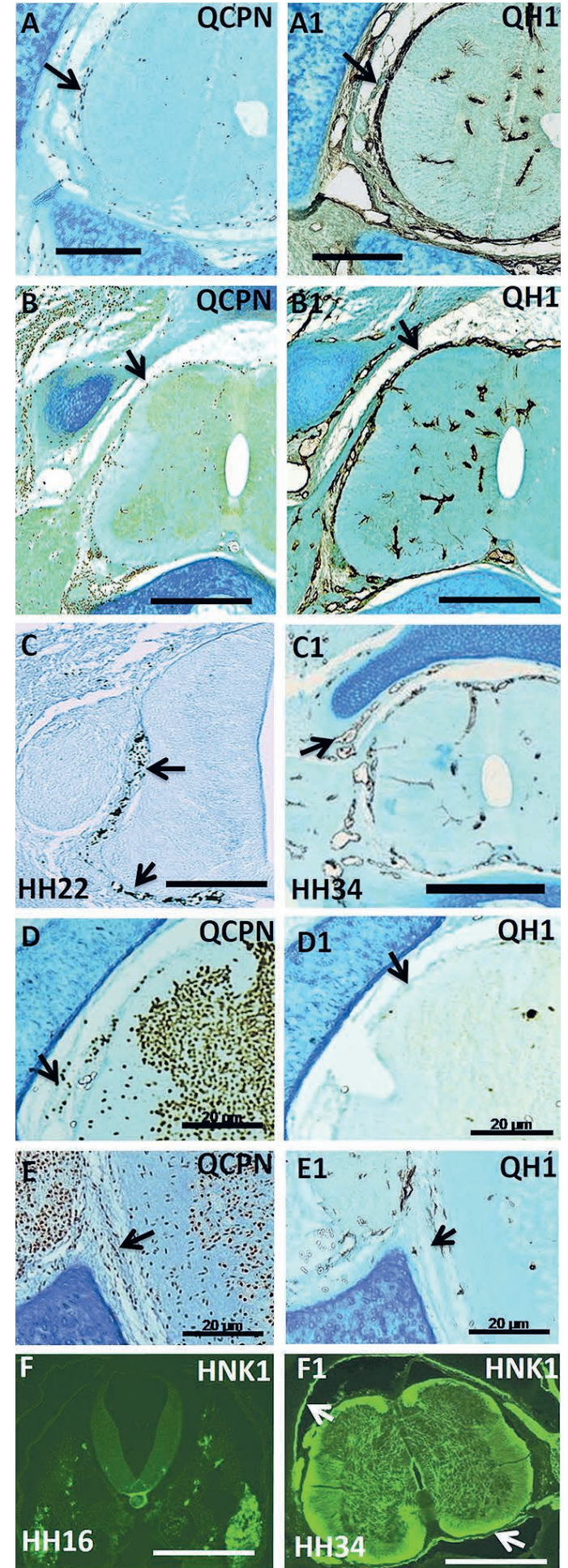

Fig. 3. Neural tube origins of chick meninges.

Quail somite transplant at cervical level (A) or thoracic level (B) resulted in the QCPN positive nuclei at the periphery of the neural tube (black arrows). Quail somites transplanted at either cervical (A1) or thoracic level (B1) gave rise to extensive vasculature development that was quail in origin identified through QH1 staining (black arrows). (C) Indian ink tracing shows vasculature t at $\mathrm{HH} 22$ and (C1) at $\mathrm{HH} 34$. Quail neural tube transplant at cervical (D) or thoracic level result in numerous cells in the meninges (black arrows) which did not develop into blood vessels (D1 and E1). (F) HNK1 expression at the cervical region at $\mathrm{HH} 16$. Note no expression around neural tube. (F1) At HH34, strong HNK1 expression in the meninges (white arrow). Scale

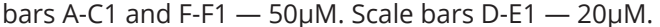

the cervical regions and 7 for the thoracic region. We found extensive populating of the meninges with cells of quail in origin at both sites (Fig. 3D and E). Furthermore we found that cells from the neural tube failed to form blood vessels (Fig. 3D1 and E1). The results show that cells from the neural tube form neck and thoracic meninges. Since the meninges develop at some distance to the neural tube we suggested that cells from this structure would have first migrated as neural crest cells before forming the meninges and should therefore express HNK1 marker associated with this tissue. We first examined the expression of this marker at a stage that precedes the formation of meninges (see Fig. 1A) and found robust expression in the dorsal root ganglion but not in any region directly associated with the meninges at HH16 (Fig. 3F1). However, when we examined the neck and thoracic region (not shown) at HH34 we found robust expression of HNK1 in the meninges (Fig. 3F1).

\section{NEURAL CREST ORIGIN OF MOUSE MENINGES}

We finally investigated the origin of mouse trunk meninges. For these experiments we turned to the Wnt1 Cre mouse which has been extensively used to identify tissues that are neural crest in origin (Chai et al., 2000; Janebodin et al., 2011; Theis et al., 2010). Relevant to this study is the fact that this line was used to show that the cranial meninges originated from neural crest (Jiang et al., 2002). Our analysis showed robust LacZ activity around the neural tube indicating the presence of neural crest cells in trunk meninges (Fig. 4A). To confirm that LacZ cells were located in the meninges we examined their positioning in relation to the expression of laminin which has been extensively used to identify this tissue (Radakovits et al., 2009). We were able to detect LacZ expression overlapping with laminin which is a key component of meninges (Fig. $4 \mathrm{~B}$ and E).

In light of the work by Jiang and colleagues 2002 and the results from this study, we propose that neural crest is the source of both head and body meninges.

\section{Discussion}

The significance of the meninges in maintaining health is ever increasing as research discovers an accumulating repertoire of roles that this tissue plays in the development and maintenance of the central nervous system. We have now moved well beyond thinking of them as only protecting the brain and spinal cord from impact damage. Recent work has shown that they are involved in the development of the CNS and contain neural stem cells that are active in all stages of higher vertebrate life (Bifari et al., 2015; Bifari et al., 2016; Borrell \& Marin, 2006). It is therefore surprising that we know so little about their development and origin. 

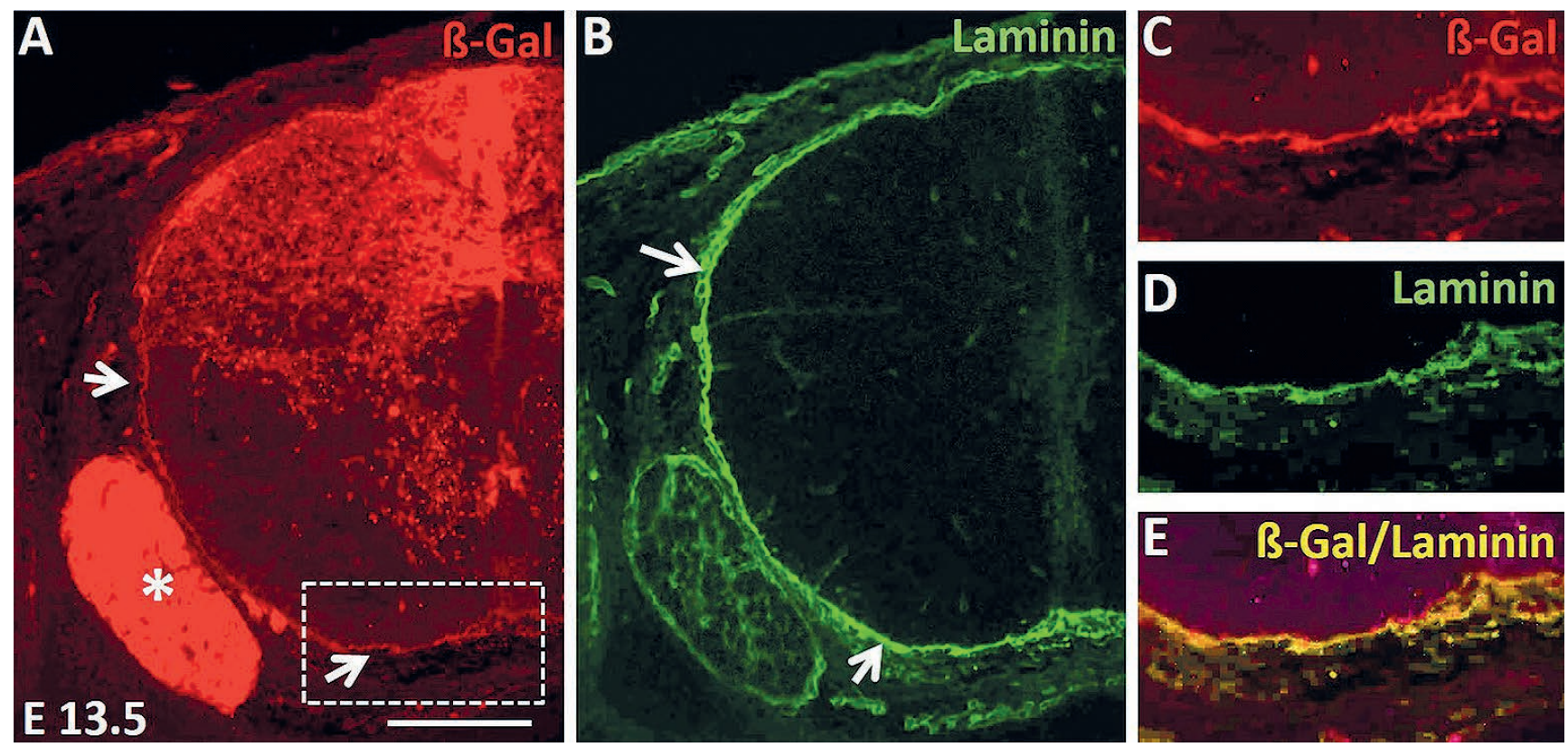

Fig. 4. Neural crest origins of mouse meninges.

(A) Cells of neural crest origin (red), identified by lineage tracing cells having expressed Wnt1 using the Wnt-1Cre/R26R lines around the entire periphery of the neural tube at the trunk level (arrows) at E13.5. Asterisk marks the dorsal root ganglion. (B) Expression of Laminin to identify meninges (arrows). (C) Detail of boxed area on (A) showing localisation of cells of neural crest origin (red) and (D) laminin to identify the meninges. (E) Co-expression of neural crest marker and laminin in meninges (yellow). Scale bars $-50 \mu \mathrm{M}$.

We started by examining the temporal development of the meninges in both the chick and the mouse. Our results show that in the chick, the meninges first appear in the body at $\mathrm{HH} 20$ in the ventral aspect of the neural tube, completely surround it by $\mathrm{HH} 26$ and form multiple layers by HH36. In the mouse these landmarks are reached at E10.5, E11.5 and E15.5 respectively. It is interesting to compare these temporal points in the two organisms and relate them to their relative stage of development. Martin correlated the development of the chick and mouse embryo and formulated a comparative scheme of tissue formation with time (Martin, 1990). Using this scheme we find that the initiation, full neural tube coverage and specialisation of the meninges in chick and mice take place at almost the same relative stages of their development.

The timing of meninges formation was determined in our study and we showed that in the trunk region they developed at HH20 and E10.5 in the chick and mouse respectively. This is highly relevant when viewed in the context of neural crest development. The temporal development of trunk neural crest in region investigated here shows that it starts at approximately HH13-14 in the chick and E9-9.5 in the mouse (Gammill and Roffers-Agarwal, 2010; Serbedzija, Bronner-Fraser, and Fraser, 1989; Serbedzija, Fraser, and Bronner-Fraser, 1990; Theveneau and Mayor, 2012). Therefore the formation of the meninges occurs soon after the migration event, undoubtedly influenced by their proximity compared to their site of origin.
The origin of the meninges has been much debated during the past century. While it is general accepted that the cranial meninges are of neural crest origin, the source of their spinal counterparts was proposed by many to be somitic (Bagnall et al., 1989; Christ et al., 2007; Nimmagadda et al., 2004). Our data disproves the later view but also offers an explanation why this incorrect view arose. We show that in both the chick and mouse, the spinal meninges are neural crest in origin. Neural crest also forms the boundary cap cells that lie adjacent to the neural tube in the trunk region (Maro et al., 2004). Therefore it is possible that the quail cells from the transplanted neural tube form this population. However the localisation of quail cells both dorsal and ventral to the neural tube, argue against this as boundary cap cells are not located at these sites.

We show that the somites make a major contribution to the vasculature of the meninges. We believe that the idea of somitic origin of spinal meninges arose because cell tracing experiments could not resolve the differing cell types that constitute the meninges (Bagnall et al., 1989). We show here that somitic cells that populate the neural tube are endothelial cells based on their co-expression of QH1. Therefore our work demonstrates that somitic cells contribute to the vasculature of the neural tube which in the past has been taken to be the meninges. However in mitigation of studies that suggested a somitic origin for meninges, they were performed at a time when tissue-specific markers were not 
so well developed and the full repertoire of somitic tissues not appreciated. Our study fully supports the notion that the somites and in particular the sclerotome give rise to the vasculature of the meninges (Halata et al., 1990; Nimmagadda et al., 2005; Nimmagadda et al., 2004). Although beyond the scope of this project, future experiments are planned, using a genetic approach through the deployment of sclerotome specific reporter lines (e.g., driven by a Paxl promoter), to prove that blood vessels of the mouse neural tube originate from this mesodermal compartment.

An interesting area for future work would be to determine the signalling events that initiate the differentiation of neural crest into meninges and whether like their cranial counter parts, they contain undifferentiated cells that are able to form neural tissues. Long term lineage tracing may give clues about the fate repertoire of neural crest cells in the meningeal region. It is conceivable that in addition to contributing to meninges that they could differentiate into pericytes, as found in the brain (Dupin and Sommer, 2012) or even neural stem cells. These studies were beyond the scope of our present investigation. Another way to address the same issue is to take advantage of technical developments in particular single cell transcriptional analysis (RNAseq) to address these issues (Shembekar, Chaipan, Utharala, and Merten, 2016). In future we aim to perform RNAseq on neural crest cells that are about to form the meninges and identify molecular footprints of signalling pathways and compare them to the transcriptional profile of early and late stage neural crest cells. We suggest it is essential to determine whether spinal meninges contain neural progenitors during either development and/or in adult life. This could be achieved by exploiting the findings of elegant studies performed in the brain which showed that cells positive to Nestin are a convenient way to identify neural progenitors. If these cells are present in the spinal meninges then their fate can be again followed by single cell RNAseq analysis (Bifari et al., 2015; Bifari et al., 2016).

In summary our data show that the meninges of the neck and trunk are neural crest in origin. These results are concordant with the results of Jiang et al. (2002) who found that cranial meninges are derived from neural crest. Therefore all meninges originate from neural crest and represent a vertebrate innovation (Shimeld and Holland, 2000).

\section{Acknowledgements}

We thank Dr Sajjida Jaffer for experimental assistance. We are indebted to Dr Anthony Otto for his assistance in preparing this manuscript as well as two anonymous reviewers for their comments that have greatly improved this work.

\section{References}

Bagnall, K. M., Higgins, S. J., and Sanders, E. J. (1989). The contribution made by cells from a single somite to tissues within a body segment and assessment of their integration with similar cells from adjacent segments. Development, 107(4), 931-943.

Bifari, F., Berton, V., Pino, A., Kusalo, M., Malpeli, G., Di Chio, M., ... Decimo, I. (2015). Meninges harbor cells expressing neural precursor markers during development and adulthood. Frontiers in Cellular Neuroscience, 9, 383. doi:10.3389/fncel.2015.00383

Bifari, F., Decimo, I., Pino, A., Llorens-Bobadilla, E., Zhao, S., Lange, C., ... Carmeliet, P. (2016). Neurogenic Radial Glialike Cells in Meninges Migrate and Differentiate into Functionally Integrated Neurons in the Neonatal Cortex. Cell Stem Cell. doi:10.1016/j.stem.2016.10.020

Bloom, W., and Fawcett, D. G. (1968). A Textbook of Histology. Philadelphia: Saunders.

Borrell, V., and Marin, O. (2006). Meninges control tangential migration of hem-derived Cajal-Retzius cells via CXCL12/ CXCR4 signaling. Nature Neuroscience, 9(10), 1284-1293. doi:10.1038/nn1764

Chai, Y., Jiang, X., Ito, Y., Bringas, P., Jr., Han, J., Rowitch, D. H., ... Sucov, H. M. (2000). Fate of the mammalian cranial neural crest during tooth and mandibular morphogenesis. Development, 127(8), 1671-1679.

Chapman, S. C., Collignon, J., Schoenwolf, G. C., and Lumsden, A. (2001). Improved method for chick wholeembryo culture using a filter paper carrier. Developmental Dynamics, 220(3), 284-289. doi:10.1002/10970177(20010301)220:3<284::AID-DVDY1102>3.0.CO;2-5

Christ, B., Huang, R., \& Scaal, M. (2007). Amniote somite derivatives. Developmental Dynamics, 236(9), 2382-2396. doi:10.1002/dvdy.21189

Clarke, A. G. (1944). The Anatomy of the Meninges. Postgraduate Medical Journal, 20(220), 74-78.

Dupin, E., and Sommer, L. (2012). Neural crest progenitors and stem cells: from early development to adulthood. Developmental Biology, 366(1), 83-95. doi:10.1016/j.ydbio.2012.02.035

Gammill, L. S., and Roffers-Agarwal, J. (2010). Division of labor during trunk neural crest development. Developmantal Biology, 344(2), 555-565. doi:10.1016/j.ydbio.2010.04.009

Haines, D. E., Harkey, H. L., and al-Mefty, O. (1993). The "subdural" space: a new look at an outdated concept. Neurosurgery, 32(1), 111-120.

Halata, Z., Grim, M., and Christ, B. (1990). Origin of spinal cord meninges, sheaths of peripheral nerves, and cutaneous receptors including Merkel cells. An experimental and ultrastructural study with avian chimeras. Anatomy and Embryology (Berl), 182(6), 529-537.

Hamburger, V., and Hamilton, H. L. (1951). A series of normal stages in the development of the chick embryo. Journal of Morphology, 88(1), 49-92.

Janebodin, K., Horst, O. V., leronimakis, N., Balasundaram, G., Reesukumal, K., Pratumvinit, B., and Reyes, M. (2011). Isolation and characterization of neural crest-derived stem cells from dental pulp of neonatal mice. PLoS One, 6(11), e27526. doi:10.1371/journal.pone.0027526

Jiang, X., Iseki, S., Maxson, R. E., Sucov, H. M., and MorrissKay, G. M. (2002). Tissue origins and interactions in the mammalian skull vault. Developmantal Biology, 241(1), 106-116. doi:10.1006/dbio.2001.0487

Kapoor, K. G., Katz, S. E., Grzybowski, D. M., and Lubow, M. (2008). Cerebrospinal fluid outflow: an evolving perspective. Brain Research Bulletin, 77(6), 327-334. doi:10.1016/j. brainresbull.2008.08.009 
Korn, J., Christ, B., and Kurz, H. (2002). Neuroectodermal origin of brain pericytes and vascular smooth muscle cells. Journal of Comparative Neurology, 442(1), 78-88. doi:10.1002/cne.1423 [pii]

Maro, G.S., Vermeren, M., Voiculescu, O., Melton, L., Cohen, J., Charnay, P., and Topilko, P. (2004). Neural crest boundary cap cells constitute a source of neuronal and glial cells of the PNS. Nature Neuroscience, 7(9), 930-938. doi:10.1038/nn1299

Martin, P. (1990). Tissue patterning in the developing mouse limb. International Journal of Developmental Biology, 34(3), 323-336.

Nimmagadda, S., Geetha Loganathan, P., Huang, R., Scaal, M., Schmidt, C., and Christ, B. (2005). BMP4 and noggin control embryonic blood vessel formation by antagonistic regulation of VEGFR-2 (Quek1) expression. Developmental Biology, 280(1), 100-110. doi:10.1016/j.ydbio.2005.01.005

Nimmagadda, S., Loganathan, P. G., Wilting, J., Christ, B., and Huang, R. (2004). Expression pattern of VEGFR-2 (Quek1) during quail development. Anatomy and Embryology (Berl), 208(3), 219-224. doi:10.1007/s00429-004-0396-z

O'Rahilly, R., and Muller, F. (1986). The meninges in human development. Journal of Neuropathology and Experimental Neurology, 45(5), 588-608.

Petricevic, J., Forempoher, G., Ostojic, L., Mardesic-Brakus, S., Andjelinovic, S., Vukojevic, K., and Saraga-Babic, M. (2011). Expression of nestin, mesothelin and epithelial membrane antigen (EMA) in developing and adult human meninges and meningiomas. Acta Histochemica, 113(7), 703-711. doi:10.1016/j.acthis.2010.09.005

Radakovits, R., Barros, C.S., Belvindrah, R., Patton, B., and Muller, U. (2009). Regulation of radial glial survival by sig- nals from the meninges. Journal of Neuroscience, 29(24), 7694-7705. doi:10.1523/JNEUROSCI.5537-08.2009

Richtsmeier, J. T., and Flaherty, K. (2013). Hand in glove: brain and skull in development and dysmorphogenesis. Acta Neuropathologica, 125(4), 469-489. doi:10.1007/s00401013-1104-y

Serbedzija, G. N., Bronner-Fraser, M., and Fraser, S. E. (1989). A vital dye analysis of the timing and pathways of avian trunk neural crest cell migration. Development, 106(4), 809-816.

Serbedzija, G. N., Fraser, S. E., and Bronner-Fraser, M. (1990). Pathways of trunk neural crest cell migration in the mouse embryo as revealed by vital dye labelling. Development, 108(4), 605-612.

Shembekar, N., Chaipan, C., Utharala, R., and Merten, C. A. (2016). Droplet-based microfluidics in drug discovery, transcriptomics and high-throughput molecular genetics. Lab on a Chip, 16(8), 1314-1331. doi:10.1039/ c6lc00249h

Shimeld, S. M., and Holland, P. W. (2000). Vertebrate innovations. Proceedings of the National Academy of Sciences U S A, 97(9), 4449-4452.

Standring, S., Ellis, H., and Wigley, C. (2005). Gray's anatomy: the anatomical basis of clinical practice. New York: Elsevier Churchill Livingstone.

Theis, S., Patel, K., Valasek, P., Otto, A., Pu, Q., Harel, I., ... Huang, R. (2010). The occipital lateral plate mesoderm is a novel source for vertebrate neck musculature. Development, 137(17), 2961-2971. doi:10.1242/dev.049726

Theveneau, E., and Mayor, R. (2012). Neural crest delamination and migration: from epithelium-to-mesenchyme transition to collective cell migration. Developmental Biology, 366(1), 34-54. doi:10.1016/j.ydbio.2011.12.041 